\title{
A modified regularization method for an inverse heat conduction problem with only boundary value
}

\author{
Wei Cheng ${ }^{1 *}$ and Yun-Jie Ma
}

"Correspondence:
chwei2007@163.com
${ }^{1}$ College of Science, Henan
University of Technology,
Zhengzhou, 450001, P.R. China
Full list of author information is
available at the end of the article

*Correspondence:

Full list of author information is

available at the end of the article

\begin{abstract}
This paper aims to solve an inverse heat conduction problem with only boundary value in a bounded domain, where the boundary data is given for $x=0$. The solution is sought in the interval $0<x \leq 1$. The problem is seriously ill posed in the Hadamard sense. Using the Hölder inequality and some inequalities, a conditional stability is proved for this problem. A modified Tikhonov regularization method is proposed to recover the stability of the solution. An order optimal error estimate between the approximate solution and the exact solution is obtained with a suitable choice of regularization parameter. Numerical results are presented to illustrate the accuracy and efficiency of the proposed method.
\end{abstract}

MSC: 65M30; 35R25; 35R30

Keywords: ill-posed problem; inverse heat conduction problem; regularization; error estimate

\section{Introduction}

In this paper we consider the following inverse heat conduction problem with only boundary value:

$$
\begin{aligned}
& u_{t}=u_{x x}, \quad 0<x<1,0<t<2 \pi, \\
& u(0, t)=f(t), \quad 0 \leq t \leq 2 \pi, \\
& u_{x}(0, t)=g(t), \quad 0 \leq t \leq 2 \pi,
\end{aligned}
$$

where $f$ and $g$ are given. This problem is ill posed [1]. We want to recover the temperature distribution $u(x, \cdot)$ for $0<x \leq 1$ from the boundary data $f$ and $g$.

The inverse heat conduction problem (IHCP) arises from many physical and engineering disciplines. It is well known that the problem is severely ill posed in the Hadamard sense that the solution (if it exists) does not depend continuously on the given data, i.e., a small measurement error in the given data can cause an enormous error in the solution [2-4]. To overcome such difficulties, some regularization techniques are required [5]. The IHCP has been considered by many authors using different methods. These methods include the wavelet and wavelet-Galerkin method [6-9], the Tikhonov method [10], the

(c) 2016 Cheng and Ma. This article is distributed under the terms of the Creative Commons Attribution 4.0 International License (http://creativecommons.org/licenses/by/4.0/), which permits unrestricted use, distribution, and reproduction in any medium, provided you give appropriate credit to the original author(s) and the source, provide a link to the Creative Commons license, and indicate if changes were made. 
mollification method [11-13], the fundamental solution method [14], the Fourier method [15], and so on.

To the best of the knowledge of the authors, the results available in the literature are mainly devoted to the IHCP with known initial-boundary value. However, in practical real-life problems we cannot know the initial condition because the heat process has already started before we estimate the problem. A few works are developed for the IHCP without initial value [1, 16]. Ginsberg [17] used a cutoff method for an IHCP with only boundary value and gave a Hölder type error estimate. Recently, Liu and Wei [18] used a quasi-reversibility regularization method for solving an IHCP without initial data. Yang and Fu [19] applied a simplified Tikhonov regularization method for determining the heat source. In this paper, we will use a modified Tikhonov regularization method to deal with the IHCP without initial value (1.1) and obtain an order optimal error estimate between the approximate solution and the exact solution.

The paper is organized as follows. In Section 2, we give the formulation of the solution for problem (1.1) and present some preliminary results. In Section 3, we prove the conditional stability for the IHCP (1.1) by using the Hölder inequality. Section 4 proposes a modified Tikhonov regularization method. An order optimal error estimate for the approximate solution is obtained with a suitable choice of regularization parameter. To verify the efficiency and accuracy of the proposed method for problem (1.1), we give two numerical examples in Section 5. A brief conclusion is given in Section 6.

\section{Mathematical formulation and preliminaries}

Throughout this paper, we use the following formulation and lemmas. For the IHCP (1.1), we want to determine the temperature distribution $u(x, \cdot)$ for $0<x \leq 1$ from the Cauchy data $f$ and $g$. Since the Cauchy data $f$ and $g$ are measured, there will be measurement errors, and we would actually have measured Cauchy data $f^{\delta}, g^{\delta} \in L^{2}[0,2 \pi]$, for which

$$
\left\|f-f^{\delta}\right\| \leq \delta, \quad\left\|g-g^{\delta}\right\| \leq \delta
$$

where the constant $\delta>0$ represents a bound on the measurement error, $\|\cdot\|$ and $(\cdot, \cdot)$ denote the norm and inner product on $L^{2}[0,2 \pi]$, respectively.

In the following, we split the IHCP (1.1) into two independent IHCPs:

$$
\begin{aligned}
& v_{t}=v_{x x}, \quad 0<x<1,0<t<2 \pi, \\
& v(0, t)=f(t), \quad 0 \leq t \leq 2 \pi \\
& v_{x}(0, t)=0, \quad 0 \leq t \leq 2 \pi
\end{aligned}
$$

and

$$
\begin{aligned}
& w_{t}=w_{x x}, \quad 0<x<1,0<t<2 \pi \\
& w(0, t)=0, \quad 0 \leq t \leq 2 \pi \\
& w_{x}(0, t)=g(t), \quad 0 \leq t \leq 2 \pi .
\end{aligned}
$$

Let $v(x, t)$ and $w(x, t)$ be the solution of problems (2.2) and (2.3), respectively. Then $u=$ $v+w$ is the solution of problem (1.1). Therefore, we only need solve problems (2.2) and (2.3), respectively. 
By the method of separation of variables, the exact solutions of problems (2.2) and (2.3) are given by

$$
v(x, t)=\sum_{n=-\infty}^{+\infty}\left(f(t), e^{i n t}\right) e^{i n t} \cosh (\sqrt{i n} x)
$$

and

$$
w(x, t)=\sum_{n=-\infty}^{+\infty} \frac{1}{\sqrt{i n}}\left(g(t), e^{i n t}\right) e^{i n t} \sinh (\sqrt{i n} x) .
$$

Then the exact solution of problem (1.1) is given by

$$
u(x, t)=\sum_{n=-\infty}^{+\infty}\left[\left(f(t), e^{i n t}\right) e^{i n t} \cosh (\sqrt{i n} x)+\frac{\left(g(t), e^{i n t}\right)}{\sqrt{i n}} e^{i n t} \sinh (\sqrt{i n} x)\right]
$$

We assume also that there exists an a priori condition for problem (1.1):

$$
\max \left\{\|v(1, \cdot)\|_{p},\|w(1, \cdot)\|_{p}\right\} \leq E, \quad p \geq 0
$$

where $\|v(1, \cdot)\|_{p}=\left\|\sum_{n=-\infty}^{+\infty}\left(1+n^{2}\right)^{p / 2}\left(v(1, \cdot), e^{i n(\cdot)}\right) e^{i n(\cdot)}\right\|$.

In order to give an error estimate for the regularized solution, we need the following lemma whose proof is similar to that of Lemma 3.2 in [20].

Lemma 2.1 Let $0<x \leq 1,0<2 \alpha<1 / e^{\sqrt[4]{3}}$. We have the following inequalities:

$$
\begin{aligned}
& \sup _{s \geq 0} \frac{e^{x s}}{1+\alpha^{2} e^{2 s}} \leq \alpha^{-x} \\
& \sup _{s \geq 0} \frac{e^{(1+x) s}\left(1+s^{4}\right)^{-\frac{p}{2}}}{1+\alpha^{2} e^{2 s}} \leq \alpha^{-(1+x)}(-\ln (2 \alpha))^{-\frac{2 p}{2 p+1}} .
\end{aligned}
$$

We need also the following results.

Lemma 2.2 Let $0<x \leq 1$, then there holds [18]:

$$
\begin{aligned}
& \lim _{n \rightarrow 0} \frac{\sinh (\sqrt{i n} x)}{\sqrt{i n}}=x, \quad\left|\frac{\sinh (\sqrt{i n} x)}{\sqrt{i n}}\right| \leq \sqrt{2} x e^{\sqrt{\frac{|n|}{2}} x}, \quad n \in \mathbb{Z}, \\
& |\cosh (\sqrt{i n} x)| \leq e^{\sqrt{\frac{|n|}{2}} x}, \quad|\sinh (\sqrt{i n} x)| \leq e^{\sqrt{\frac{|n|}{2}} x}, \quad n \in \mathbb{Z}, \\
& |\cosh (\sqrt{i n})| \geq c e^{\sqrt{\frac{|n|}{2}}}, \quad|\sinh (\sqrt{i n})| \geq c e^{\sqrt{\frac{|n|}{2}}}, \quad|n| \in \mathbb{N}^{+},
\end{aligned}
$$

where $c=\left(1-e^{-\sqrt{2}}\right) / 2$.

\section{Conditional stability}

In this section, we will provide the conditional stabilities for problems (2.2), (2.3), and (1.1), respectively. 
Theorem 3.1 Let the a priori bound (2.7) hold and $v(x, t)$ be the solution of problem (2.2) given by (2.4) with the exact data $f(t)$, then for a fixed $x \in(0,1)$ the following estimate holds:

$$
\|v(x, \cdot)\| \leq c^{-x} E^{x}\|f\|^{1-x}
$$

Proof By the Hölder inequality and (2.4), we have

$$
\begin{aligned}
\|v(x, \cdot)\|^{2} & =\left\|\sum_{n=-\infty}^{+\infty} \cosh (\sqrt{i n} x)\left(f, e^{i n(\cdot)}\right) e^{i n(\cdot)}\right\|^{2}=\sum_{n=-\infty}^{+\infty}|\cosh (\sqrt{i n} x)|^{2}\left|f_{n}\right|^{2} \\
& =\sum_{n=-\infty}^{+\infty}\left(|\cosh (\sqrt{i n} x)|^{2}\left|f_{n}\right|^{2 x}\right)\left|f_{n}\right|^{2(1-x)} \\
& \leq\left[\sum_{n=-\infty}^{+\infty}\left(|\cosh (\sqrt{i n} x)|^{2}\left|f_{n}\right|^{2 x}\right)^{\frac{1}{x}}\right]^{x}\left[\sum_{n=-\infty}^{+\infty}\left(\left|f_{n}\right|^{2(1-x)}\right)^{\frac{1}{1-x}}\right]^{1-x} \\
& =\left[\sum_{n=-\infty}^{+\infty}|\cosh (\sqrt{i n} x)|^{\frac{2}{x}}\left|f_{n}\right|^{2}\right]^{x}\left[\sum_{n=-\infty}^{+\infty}\left|f_{n}\right|^{2}\right]^{1-x} \\
& =\left[\sum_{n=-\infty}^{+\infty}|\cosh (\sqrt{i n} x)|^{\frac{2}{x}}|\cosh (\sqrt{i n})|^{-2}\left|\left(v(1, \cdot), e^{i n(\cdot)}\right)\right|^{2}\right]^{x}\|f\|^{2(1-x)} \\
& \leq\left[\sum_{n=-\infty}^{+\infty}|\cosh (\sqrt{i n} x)|^{\frac{2}{x}}|\cosh (\sqrt{i n})|^{-2}\left(1+n^{2}\right)^{p}\left|\left(v(1, \cdot), e^{i n(\cdot)}\right)\right|^{2}\right]^{x}\|f\|^{2(1-x)} \\
& \leq \max _{n \in \mathbb{Z}}\left[|\cosh (\sqrt{i n} x)|^{2}|\cosh (\sqrt{i n})|^{-2 x}\right]\|v(1, \cdot)\|_{p}^{2 x}\|f\|^{2(1-x)} \cdot
\end{aligned}
$$

Using (2.11) and (2.12), we have

$$
|\cosh (\sqrt{\text { in } x})|^{2}|\cosh (\sqrt{\text { in }})|^{-2 x} \leq\left(e^{\sqrt{|n| / 2} x}\right)^{2}\left(c e^{\sqrt{|n| / 2}}\right)^{-2 x}=c^{-2 x}, \quad|n| \in \mathbb{N}^{+},
$$

then we get

$$
\max _{n \in \mathbb{Z}}\left[|\cosh (\sqrt{i n} x)|^{2}|\cosh (\sqrt{i n})|^{-2 x}\right]=\max _{n \in \mathbb{Z}}\left\{1, \max _{n \in \mathbb{N}^{+}} \frac{|\cosh (\sqrt{i n} x)|^{2}}{|\cosh (\sqrt{i n})|^{2 x}}\right\} \leq c^{-2 x} .
$$

Combining with the a priori bound (2.7), we obtain

$$
\|v(x, \cdot)\|^{2} \leq c^{-2 x} E^{2 x}\|f\|^{2(1-x)} .
$$

The proof is completed.

Remark 3.2 If $v_{1}(x, t)$ and $v_{2}(x, t)$ are the solutions of problem (2.2) with the exact data $f_{1}(t)$ and $f_{2}(t)$, respectively, then for a fixed $x \in(0,1)$ we have

$$
\left\|v_{1}(x, \cdot)-v_{2}(x, \cdot)\right\| \leq c^{-x} E^{x}\left\|f_{1}(\cdot)-f_{2}(\cdot)\right\|^{1-x} .
$$

Similarly, we have the following results. 
Theorem 3.3 Suppose that $w(x, t)$ is the solution of problem (2.3) given by (2.5) with the exact data $g(t)$ and the a priori bound $(2.7)$ is valid, then for a fixed $x \in(0,1)$ the following estimate holds:

$$
\|w(x, \cdot)\| \leq c^{-x}(\sqrt{2} x)^{1-x} E^{x}\|g\|^{1-x} .
$$

Proof Using the Hölder inequality and (2.5), we have

$$
\begin{aligned}
\|w(x, \cdot)\|^{2} & =\left|\sum_{n=-\infty}^{+\infty} \frac{\sinh (\sqrt{i n} x)}{\sqrt{i n}}\left(g, e^{i n(\cdot)}\right) e^{i n(\cdot)}\right|^{2}=\sum_{n=-\infty}^{+\infty}\left|\frac{\sinh (\sqrt{i n} x)}{\sqrt{i n}}\right|^{2}\left|g_{n}\right|^{2} \\
& =\sum_{n=-\infty}^{+\infty}\left(|(\sinh (\sqrt{i n} x)) / \sqrt{i n}|^{2}\left|g_{n}\right|^{2 x}\right)\left|g_{n}\right|^{2(1-x)} \\
& \leq\left[\sum_{n=-\infty}^{+\infty}\left(|(\sinh (\sqrt{i n} x)) / \sqrt{i n}|^{2}\left|g_{n}\right|^{2 x}\right)^{\frac{1}{x}}\right]^{x}\left[\sum_{n=-\infty}^{+\infty}\left|g_{n}\right|^{2}\right]^{1-x} \\
& =\left[\sum_{n=-\infty}^{+\infty}\left|\frac{\sinh (\sqrt{i n} x)}{\sqrt{i n}}\right|^{\frac{2}{x}}\left|\frac{\sinh (\sqrt{i n})}{\sqrt{i n}}\right|^{-2}\left|\left(w(1, \cdot), e^{i n(\cdot)}\right)\right|^{2}\right]^{x}\|g\|^{2(1-x)} \\
& \leq\left[\sum_{n=-\infty}^{+\infty}\left|\frac{\sinh (\sqrt{i n} x)}{\sqrt{i n}}\right|^{\frac{2}{x}}\left|\frac{\sinh (\sqrt{i n})}{\sqrt{i n}}\right|^{-2}\left(1+n^{2}\right)^{p}\left|\left(w(1, \cdot), e^{i n(\cdot)}\right)\right|^{2}\right]^{x}\|g\|^{2(1-x)} \\
& \leq \max _{n \in \mathbb{Z}}\left[\left|\frac{\sinh (\sqrt{i n} x)}{\sqrt{i n}}\right|^{2}\left|\frac{\sinh (\sqrt{i n})}{\sqrt{i n}}\right|^{-2 x}\right]\|w(1, \cdot)\|_{p}^{2 x}\|g\|^{2(1-x)} \cdot
\end{aligned}
$$

From (2.10)-(2.12), we get

$$
\begin{aligned}
\left|\frac{\sinh (\sqrt{i n} x)}{\sqrt{i n}}\right|^{2}\left|\frac{\sinh (\sqrt{i n})}{\sqrt{i n}}\right|^{-2 x} & =\left|\frac{\sinh (\sqrt{i n} x)}{\sqrt{i n}}\right|^{2(1-x)}\left|\frac{\sinh (\sqrt{i n} x)}{\sinh (\sqrt{i n})}\right|^{2 x} \\
& \leq\left(\sqrt{2} x e^{\sqrt{|n| / 2} x}\right)^{2(1-x)}\left(\frac{e^{\sqrt{|n| / 2} x}}{c e^{\sqrt{|n| / 2}}}\right)^{2 x} \\
& =c^{-2 x}(\sqrt{2} x)^{2(1-x)}, \quad|n| \in \mathbb{N}^{+}
\end{aligned}
$$

so

$$
\begin{aligned}
\max _{n \in \mathbb{Z}}\left[\left|\frac{\sinh (\sqrt{i n} x)}{\sqrt{i n}}\right|^{2}\left|\frac{\sinh (\sqrt{i n})}{\sqrt{i n}}\right|^{-2 x}\right] & =\max _{n \in \mathbb{Z}}\left\{x^{2}, \max _{n \in \mathbb{N}^{+}} \frac{|(\sinh (\sqrt{i n} x)) / \sqrt{i n}|^{2}}{|(\sinh (\sqrt{i n})) / \sqrt{i n}|^{2 x}}\right\} \\
& \leq c^{-2 x}(\sqrt{2} x)^{2(1-x)} .
\end{aligned}
$$

Combining with (2.7), we obtain

$$
\|w(x, \cdot)\|^{2} \leq c^{-2 x}(\sqrt{2} x)^{2(1-x)} E^{2 x}\|g\|^{2(1-x)} .
$$

Estimate (3.3) is proved.

Remark 3.4 If $w_{1}(x, t)$ and $w_{2}(x, t)$ are the solutions of problem (2.3) with the exact data $g_{1}(t)$ and $g_{2}(t)$, respectively, then for a fixed $x \in(0,1)$ we have

$$
\left\|w_{1}(x, \cdot)-w_{2}(x, \cdot)\right\| \leq c^{-x}(\sqrt{2} x)^{(1-x)} E^{x}\left\|g_{1}(\cdot)-g_{2}(\cdot)\right\|^{1-x} .
$$


From Theorems 3.1 and 3.3, we then obtain the following theorem.

Theorem 3.5 Let the a priori bound (2.7) hold and $u(x, t)$ be the solution of problem (1.1) given by (2.6) with the exact data $f(t)$ and $g(t)$, then for a fixed $x \in(0,1)$ we have the following estimate:

$$
\|u(x, \cdot)\| \leq c^{-x} E^{x}\|f\|^{1-x}+c^{-x}(\sqrt{2} x)^{1-x} E^{x}\|g\|^{1-x} .
$$

Remark 3.6 If $u_{1}(x, t)$ and $u_{2}(x, t)$ are the solutions of problem (1.1) with the exact data pairs $\left[f_{1}(t), g_{1}(t)\right]$ and $\left[f_{2}(t), g_{2}(t)\right]$, respectively, then for a fixed $x \in(0,1)$ we get

$$
\left\|u_{1}(x, \cdot)-u_{2}(x, \cdot)\right\| \leq c^{-x} E^{x}\left\|f_{1}(\cdot)-f_{2}(\cdot)\right\|^{1-x}+c^{-x}(\sqrt{2} x)^{(1-x)} E^{x}\left\|g_{1}(\cdot)-g_{2}(\cdot)\right\|^{1-x} .
$$

\section{Regularization and error estimates}

Since Cauchy problems (2.2) and (2.3) are all severely ill posed, we should apply a regularization method to solve them.

\subsection{Regularization and error estimate for problem (2.2)}

For problem (2.2), we define an operator $K: v(x, \cdot) \rightarrow f(\cdot)$, then problem (2.2) can be rewritten as the following operator equation:

$$
K v(x, t)=f(t), \quad 0<x \leq 1 .
$$

Combining with equation (2.4), we have

$$
K v(x, t)=\sum_{n=-\infty}^{+\infty}\left(v(x, t), e^{i n t}\right)(\cosh (\sqrt{i n} x))^{-1} e^{i n t} .
$$

Consequently, $K$ is an operator with eigenvalues

$$
k_{n}=(\cosh (\sqrt{i n} x))^{-1}
$$

For disturbed data $f^{\delta}(t)$, we use the Tikhonov regularization method, which seeks a function $v_{\alpha}^{\delta}(x, \cdot)$ from minimizing quadratic functional

$$
J_{\alpha}\left(v^{\delta}\right):=\left\|K v^{\delta}-f^{\delta}\right\|^{2}+\alpha^{2}\left\|v^{\delta}\right\|^{2}
$$

According to Theorem 2.11 of [21], this Tikhonov functional $J_{\alpha}$ has a unique minimum $v_{\alpha}^{\delta}(x, \cdot)$ which is the unique solution of the normal equation

$$
K^{*} K v_{\alpha}^{\delta}+\alpha^{2} v_{\alpha}^{\delta}=K^{*} f^{\delta}, \quad \alpha>0
$$

here $K^{*}$ is the adjoint of $K$. Using the properties of the inner product, we obtain the eigenvalues of operator $K^{*}$ :

$$
\bar{k}_{n}=\overline{(\cosh (\sqrt{i n} x))^{-1}}
$$


where the symbol $\overline{h(\cdot)}$ denotes the complex conjugate of the function $h(\cdot)$. Combining (4.2), (4.3), (4.6) with (4.5), we get

$$
\sum_{n=-\infty}^{+\infty}\left(\bar{k}_{n} k_{n}+\alpha^{2}\right)\left(v_{\alpha}^{\delta}(x, t), e^{i n t}\right) e^{i n t}=\sum_{n=-\infty}^{+\infty} \bar{k}_{n}\left(f^{\delta}(t), e^{i n t}\right) e^{i n t}
$$

This yields

$$
\begin{aligned}
v_{\alpha}^{\delta}(x, t) & =\sum_{n=-\infty}^{+\infty}\left(v_{\alpha}^{\delta}(x, t), e^{i n t}\right) e^{i n t}=\sum_{n=-\infty}^{+\infty} \frac{\bar{k}_{n}}{\left|k_{n}\right|^{2}+\alpha^{2}}\left(f^{\delta}(t), e^{i n t}\right) e^{i n t} \\
& =\sum_{n=-\infty}^{+\infty} \frac{\cosh (\sqrt{i n} x)}{1+\alpha^{2}|\cosh (\sqrt{i n} x)|^{2}}\left(f^{\delta}(t), e^{i n t}\right) e^{i n t} .
\end{aligned}
$$

We call $v_{\alpha}^{\delta}(x, t)$ given by (4.7) the Tikhonov approximate solution of problem (2.2). In order to derive the error estimate between the regularized solution and the exact solution, we replace the original filter $\frac{1}{1+\alpha^{2}|\cosh (\sqrt{i n} x)|^{2}}$ with another filter $\frac{1}{1+\alpha^{2}|\cosh (\sqrt{i n})|^{2}}$. Thus, the modified regularized solution of problem (2.2) becomes

$$
v_{\alpha}^{\delta, *}(x, t):=\sum_{n=-\infty}^{+\infty} \frac{\cosh (\sqrt{i n} x)}{1+\alpha^{2}|\cosh (\sqrt{i n})|^{2}}\left(f^{\delta}(t), e^{i n t}\right) e^{i n t}
$$

We then have an error estimate for the modified Tikhonov approximate solution of problem (2.2).

Theorem 4.1 Let $v(x, t)$ given by $(2.4)$ and $v_{\alpha}^{\delta, *}(x, t)$ given by $(4.8)$ be the exact solution and modified Tikhonov regularization solution of problem (2.2), respectively. Suppose that the noisy data $f^{\delta}(t)$ satisfies (2.1) and the a priori condition (2.7) is valid. If $0<2 \alpha<1 / e^{\sqrt[4]{3}}$ and we select the regularization parameter $\alpha$ as

$$
\alpha=(\delta / E)(\ln (E / \delta))^{\frac{2 p}{2 p+1}}
$$

then for a fixed $x \in(0,1]$, we have the following stability estimate:

$$
\left\|v(x, \cdot)-v_{\alpha}^{\delta, *}(x, \cdot)\right\| \leq c^{-x} E^{x} \delta^{1-x}\left(\ln \frac{E}{\delta}\right)^{\frac{-2 p}{2 p+1} x}\left[1+c^{-1}+o(1)\right], \quad \delta \rightarrow 0
$$

Proof By using the triangle inequality, with (2.4) and (4.8), we have

$$
\begin{aligned}
\left\|v(x, \cdot)-v_{\alpha}^{\delta, *}(x, \cdot)\right\| & =\left\|\sum_{n=-\infty}^{+\infty} k_{n}^{-1}\left[\left(f, e^{i n(\cdot)}\right)-\frac{\left(f^{\delta}, e^{i n(\cdot)}\right)}{1+\alpha^{2}|\cosh (\sqrt{i n})|^{2}}\right] e^{i n(\cdot)}\right\| \\
\leq & \left\|\sum_{n=-\infty}^{+\infty} \frac{k_{n}^{-1} \alpha^{2}|\cosh (\sqrt{i n})|^{2}}{1+\alpha^{2}|\cosh (\sqrt{i n})|^{2}}\left(f, e^{i n(\cdot)}\right) e^{i n(\cdot)}\right\| \\
& +\left\|\sum_{n=-\infty}^{+\infty} \frac{k_{n}^{-1}}{1+\alpha^{2}|\cosh (\sqrt{i n})|^{2}}\left(f-f^{\delta}, e^{i n(\cdot)}\right) e^{i n(\cdot)}\right\|
\end{aligned}
$$


Ching and Ma Boundary Value Problems (2016) 2016:100

Page 8 of 14

$$
\begin{aligned}
\leq & \alpha^{2} \sup _{n \in \mathbb{Z}} \frac{|\cosh (\sqrt{i n} x) \overline{\cosh (\sqrt{i n})}|\left(1+n^{2}\right)^{-\frac{p}{2}}}{1+\alpha^{2}|\cosh (\sqrt{i n})|^{2}} \\
\times & \left\|\sum_{n=-\infty}^{+\infty}\left(1+|n|^{2}\right)^{\frac{p}{2}}\left(v(1, \cdot), e^{i n(\cdot)}\right) e^{i n(\cdot)}\right\| \\
& +\sup _{n \in \mathbb{Z}} \frac{|\cosh (\sqrt{i n} x)|}{1+\alpha^{2}|\cosh (\sqrt{i n})|^{2}}\left\|\sum_{n=-\infty}^{+\infty}\left(f-f^{\delta}, e^{i n(\cdot)}\right) e^{i n(\cdot)}\right\| \\
= & \alpha^{2} \sup _{n \in \mathbb{Z}} A(n)\|v(1, \cdot)\|_{p}+\sup _{n \in \mathbb{Z}} B(n)\left\|f-f^{\delta}\right\|,
\end{aligned}
$$

where

$$
A(n)=\frac{|\cosh (\sqrt{i n} x) \overline{\cosh (\sqrt{i n})}|\left(1+n^{2}\right)^{-\frac{p}{2}}}{1+\alpha^{2}|\cosh (\sqrt{i n})|^{2}}, \quad B(n)=\frac{|\cosh (\sqrt{i n} x)|}{1+\alpha^{2}|\cosh (\sqrt{i n})|^{2}} .
$$

Let $s=\sqrt{|n| / 2}$. Using the inequalities (2.11)-(2.12) and (2.8), we can get

$$
B(n)=\frac{|\cosh (\sqrt{i n} x)|}{1+\alpha^{2}|\cosh (\sqrt{i n})|^{2}} \leq \frac{e^{\sqrt{|n| / 2} x}}{1+(c \alpha)^{2} e^{\sqrt{2|n|}}}=\frac{e^{x s}}{1+(c \alpha)^{2} e^{2 s}} \leq(c \alpha)^{-x}, \quad|n| \in \mathbb{N}^{+} .
$$

Thus, we have

$$
\begin{aligned}
\sup _{n \in \mathbb{Z}} B(n) & =\max \left\{\frac{1}{1+\alpha^{2}}, \sup _{|n| \in \mathbb{N}^{+}} \frac{|\cosh (\sqrt{i n} x)|}{1+\alpha^{2}|\cosh (\sqrt{i n})|^{2}}\right\} \\
& =\max \left\{\frac{1}{1+\alpha^{2}},(c \alpha)^{-x}\right\}=(c \alpha)^{-x} .
\end{aligned}
$$

Analogously, we can estimate $\sup _{n \in \mathbb{Z}} A(n)$. From the inequalities (2.11)-(2.12) and (2.9), we get

$$
\begin{aligned}
A(n) & =\frac{|\cosh (\sqrt{i n} x) \overline{\cosh (\sqrt{i n})}|\left(1+n^{2}\right)^{-\frac{p}{2}}}{1+\alpha^{2}|\cosh (\sqrt{i n})|^{2}} \leq \frac{e^{\sqrt{|n| / 2}(x+1)}\left(1+n^{2}\right)^{-\frac{p}{2}}}{1+(c \alpha)^{2} e^{\sqrt{2|n|}}} \\
& \leq \frac{e^{(x+1) s}\left(1+s^{4}\right)^{-\frac{p}{2}}}{1+(c \alpha)^{2} e^{2 s}} \leq(c \alpha)^{-(x+1)}\left(\ln \frac{1}{2 \alpha}\right)^{-\frac{2 p}{2 p+1}}, \quad|n| \in \mathbb{N}^{+} .
\end{aligned}
$$

Then we have

$$
\sup _{n \in \mathbb{Z}} A(n)=\max \left\{\frac{1}{1+\alpha^{2}},(c \alpha)^{-(x+1)}\left(\ln \frac{1}{2 \alpha}\right)^{-\frac{2 p}{2 p+1}}\right\}=(c \alpha)^{-(x+1)}\left(\ln \frac{1}{2 \alpha}\right)^{-\frac{2 p}{2 p+1}} .
$$

Combining with (4.11)-(4.12), conditions (2.7), (2.1) and the choice of $\alpha$ given by (4.9), we obtain

$$
\begin{aligned}
\left\|v(x, \cdot)-v_{\alpha}^{\delta, *}(x, \cdot)\right\| & \leq c^{-(x+1)} \alpha^{1-x}\left(\ln \frac{1}{2 \alpha}\right)^{-\frac{2 p}{2 p+1}} E+(c \alpha)^{-x} \delta \\
& \leq c^{-(x+1)} E\left(\frac{\delta}{E}\left(\ln \frac{E}{\delta}\right)^{\frac{2 p}{2 p+1}}\right)^{1-x}\left(\ln \left(\frac{E}{2 \delta}\left(\ln \frac{E}{\delta}\right)^{-\frac{2 p}{2 p+1}}\right)\right)^{-\frac{2 p}{2 p+1}}
\end{aligned}
$$




$$
\begin{aligned}
& +\left(\frac{c \delta}{E}\left(\ln \frac{E}{\delta}\right)^{\frac{2 p}{2 p+1}}\right)^{-x} \delta \\
= & c^{-x} E^{x} \delta^{1-x}\left(\ln \frac{E}{\delta}\right)^{\frac{-2 p}{2 p+1} x}\left[c^{-1}\left(\frac{\ln \frac{E}{\delta}}{\ln \frac{E}{2 \delta}-\frac{2 p}{2 p+1} \ln \left(\ln \frac{E}{\delta}\right)}\right)^{\frac{2 p}{2 p+1}}+1\right] .
\end{aligned}
$$

Note that $\frac{\ln (E / \delta)}{\ln \frac{E}{2 \delta}-\frac{2 p}{2 p+1} \ln \left(\ln \frac{E}{\delta}\right)} \rightarrow 1$ for $\delta \rightarrow 0$. The proof is completed.

\subsection{Regularization and error estimate for problem (2.3)}

For problem (2.3), the Tikhonov method involves minimizing the quadratic functional:

$$
\left\|T w^{\delta}-g^{\delta}\right\|^{2}+\alpha^{2}\left\|w^{\delta}\right\|^{2}
$$

where $T: w(x, \cdot) \rightarrow g(\cdot)$ is a forward operator. We know that the above Tikhonov functional has a unique minimum $w_{\alpha}^{\delta}(x, \cdot)$ which is the unique solution of the normal equation

$$
T^{*} T w_{\alpha}^{\delta}+\alpha^{2} w_{\alpha}^{\delta}=T^{*} g^{\delta}, \quad \alpha>0 .
$$

We can obtain the Tikhonov regularized solution of problem (2.3):

$$
w_{\alpha}^{\delta}(x, t)=\sum_{n=-\infty}^{+\infty} \frac{t_{n}^{-1}}{1+\alpha^{2}\left|\frac{\sinh (\sqrt{\text { in } x)}}{\sqrt{\text { in }}}\right|^{2}}\left(g^{\delta}(t), e^{i n t}\right) e^{i n t},
$$

where $t_{n}=\frac{\sqrt{i n}}{\sinh (\sqrt{\operatorname{lin} x})}$ is the eigenvalues of operator $T$. Similarly, we use the filter $\frac{1}{1+\alpha^{2} \mid \sinh \left(\left.\sqrt{\text { in })}\right|^{2}\right.}$ to replace the original filter $\frac{1}{1+\alpha^{2}\left|\frac{\sinh (\sqrt{\operatorname{in} x})}{\sqrt{\text { in }}}\right|^{2}}$. Therefore, we get the modified regularized solution of problem (2.3):

$$
w_{\alpha}^{\delta, *}(x, t)=\sum_{n=-\infty}^{+\infty} \frac{t_{n}^{-1}}{1+\alpha^{2}|\sinh (\sqrt{i n})|^{2}}\left(g^{\delta}(t), e^{i n t}\right) e^{i n t} .
$$

Theorem 4.2 Suppose that $w(x, t)$ is the exact solution given by $(2.5)$, and $w_{\alpha}^{\delta, *}(x, t)$ given by (4.16) is the modified Tikhonov regularization solution of problem (2.3). Let the noisy data $g^{\delta}(t)$ satisfy (2.1) and the a priori condition (2.7) be valid. If $0<2 \alpha<1 / e^{\sqrt[4]{3}}$ and the regularization parameter is given by (4.9). Then for a fixed $x \in(0,1]$, we have

$$
\left\|w(x, \cdot)-w_{\alpha}^{\delta, *}(x, \cdot)\right\| \leq c^{-x} E^{x} \delta^{1-x}\left(\ln \frac{E}{\delta}\right)^{\frac{-2 p}{2 p+1} x}\left[\sqrt{2} x+c^{-1}+o(1)\right], \quad \delta \rightarrow 0 .
$$

Proof By using the triangle inequality, with (2.5) and (4.16), we have

$$
\begin{aligned}
\left\|w(x, \cdot)-w_{\alpha}^{\delta, *}(x, \cdot)\right\|= & \left\|\sum_{n=-\infty}^{+\infty} t_{n}^{-1}\left[\left(g, e^{i n(\cdot)}\right)-\frac{\left(g^{\delta}, e^{i n(\cdot)}\right)}{1+\alpha^{2}|\sinh (\sqrt{i n})|^{2}}\right] e^{i n(\cdot)}\right\| \\
\leq & \alpha^{2} \sup _{n \in \mathbb{Z}} \frac{|\sinh (\sqrt{i n} x) \overline{\sinh (\sqrt{i n})}|\left(1+n^{2}\right)^{-\frac{p}{2}}}{1+\alpha^{2}|\sinh (\sqrt{i n})|^{2}} \\
& \times\left\|\sum_{n=-\infty}^{+\infty}\left(1+|n|^{2}\right)^{\frac{p}{2}}\left(w(1, \cdot), e^{i n(\cdot)}\right) e^{i n(\cdot)}\right\|
\end{aligned}
$$




$$
\begin{aligned}
& +\sup _{n \in \mathbb{Z}} \frac{|(\sinh (\sqrt{i n} x)) / \sqrt{i n}|}{1+\alpha^{2}|\sinh (\sqrt{i n})|^{2}}\left\|\sum_{n=-\infty}^{+\infty}\left(g-g^{\delta}, e^{i n(\cdot)}\right) e^{i n(\cdot)}\right\| \\
=: & \alpha^{2} \sup _{n \in \mathbb{Z}} C(n)\|w(1, \cdot)\|_{p}+\sup _{n \in \mathbb{Z}} D(n)\left\|f-f^{\delta}\right\| .
\end{aligned}
$$

Using the methods dealing with $\sup _{n \in \mathbb{Z}} A(n)$ and $\sup _{n \in \mathbb{Z}} B(n)$, with (2.10)-(2.12), (2.8), and (2.9), we obtain

$$
\sup _{n \in \mathbb{Z}} C(n) \leq(c \alpha)^{-(x+1)}\left(\ln \frac{1}{2 \alpha}\right)^{-\frac{2 p}{2 p+1}}, \quad \sup _{n \in \mathbb{Z}} D(n) \leq \sqrt{2} x(c \alpha)^{-x} .
$$

Combining with (4.18), conditions (2.7), (2.1), and the choice of $\alpha$ given by (4.9), we get

$$
\begin{aligned}
\left\|w(x, \cdot)-w_{\alpha}^{\delta, *}(x, \cdot)\right\| & \leq c^{-(x+1)} \alpha^{1-x}\left(\ln \frac{1}{2 \alpha}\right)^{-\frac{2 p}{2 p+1}} E+\sqrt{2} x(c \alpha)^{-x} \delta \\
& \leq c^{-x} E^{x} \delta^{1-x}\left(\ln \frac{E}{\delta}\right)^{\frac{-2 p}{2 p+1} x}\left[c^{-1}\left(\frac{\ln \frac{E}{\delta}}{\ln \frac{E}{2 \delta}-\frac{2 p}{2 p+1} \ln \left(\ln \frac{E}{\delta}\right)}\right)^{\frac{2 p}{2 p+1}}+\sqrt{2} x\right] .
\end{aligned}
$$

Note that $\frac{\ln (E / \delta)}{\ln \frac{E}{2 \delta}-\frac{2 p}{2 p+1} \ln \left(\ln \frac{E}{\delta}\right)} \rightarrow 1$ for $\delta \rightarrow 0$. The theorem is proved.

We give the regularized solution for problem (1.1):

$$
u_{\alpha}^{\delta}(x, t)=\sum_{n=-\infty}^{+\infty}\left[\frac{(\cosh (\sqrt{\operatorname{in} x}))\left(f^{\delta}(t), e^{i n t}\right)}{1+\alpha^{2}|\cosh (\sqrt{\operatorname{in} x})|^{2}}+\frac{\frac{\sinh (\sqrt{\operatorname{in} x})}{\sqrt{i n}}\left(g^{\delta}(t), e^{i n t}\right)}{1+\alpha^{2}\left|\frac{\sinh (\sqrt{\operatorname{in} x})}{\sqrt{i n}}\right|^{2}}\right] e^{i n t} .
$$

Similarly, the modified Tikhonov regularized solution is

$$
u_{\alpha}^{\delta, *}(x, t)=\sum_{n=-\infty}^{+\infty}\left[\frac{(\cosh (\sqrt{i n} x))\left(f^{\delta}(t), e^{i n t}\right)}{1+\alpha^{2}|\cosh (\sqrt{i n})|^{2}}+\frac{\frac{\sinh (\sqrt{i n} x)}{\sqrt{i n}}\left(g^{\delta}(t), e^{i n t}\right)}{1+\alpha^{2}|\sinh (\sqrt{i n})|^{2}}\right] e^{i n t} .
$$

Analogously, we have the error estimate for problem (1.1).

Theorem 4.3 Let $u(x, t)$ be the exact solution given by (2.6), and $u_{\alpha}^{\delta, *}(x, t)$ given by (4.20) be the modified Tikhonov regularization solution of problem (1.1). Suppose that the noisy data $f^{\delta}(t)$ and $g^{\delta}(t)$ satisfy (2.1) and the a priori condition (2.7) is valid. If $0<2 \alpha<1 / e^{\sqrt[4]{3}}$ and the regularization parameter is chosen as (4.9), then for fixed $x \in(0,1]$, we obtain

$$
\left\|u(x, \cdot)-u_{\alpha}^{\delta, *}(x, \cdot)\right\| \leq c^{-x} E^{x} \delta^{1-x}\left(\ln \frac{E}{\delta}\right)^{\frac{-2 p}{2 p+1} x}\left[\sqrt{2} x+1+2 c^{-1}+o(1)\right], \quad \delta \rightarrow 0 .
$$

\section{Remark 4.4}

(i) If we choose $p=0$, estimate (4.21) becomes

$$
\left\|u(x, \cdot)-u_{\alpha}^{\delta, *}(x, \cdot)\right\| \leq\left((\sqrt{2} x+1) c^{-x}+2 c^{-(x+1)}\right) E^{x} \delta^{1-x},
$$

it is a Hölder type stability estimate.

(ii) If we choose $p>0$, estimate (4.21) is a logarithmic-Hölder type error estimate, especially at $x=1$, it is a logarithmic type convergence estimate. 


\section{Numerical experiments}

In this section, we present two numerical examples to illustrate the effectiveness of the suggested regularization method. The grid numbers on the space and time intervals are taken to be $M=101, J=301$, refer to [18]. The noisy Cauchy data are generated by

$$
f^{\delta}\left(t_{j}\right)=f\left(t_{j}\right)(1+\varepsilon \cdot \operatorname{rand}(j)), \quad g^{\delta}\left(t_{j}\right)=g\left(t_{j}\right)(1+\varepsilon \cdot \operatorname{rand}(j)),
$$

where $t_{j}$ is a set of discrete times on interval $[0,2 \pi], f\left(t_{j}\right)$ and $g\left(t_{j}\right)$ are the exact Cauchy data, $\operatorname{rand}\left(t_{j}\right)$ is a random number uniformly distributed on $[-1,1]$, and the magnitude $\varepsilon$ indicates the relative noise level. In the tests, the noise level $\delta$ is computed according to

$$
\delta=\max \left\{\left\|f-f^{\delta}\right\|,\left\|g-g^{\delta}\right\|\right\}
$$

In order to present the performance of the modified Tikhonov method, we define the relative root mean square error at fixed $x$ as

$$
e(u)=\frac{\sqrt{\sum_{j=1}^{J}\left(u\left(\cdot, t_{j}\right)-u_{\alpha}^{\delta, *}\left(\cdot, t_{j}\right)\right)^{2}}}{\sqrt{\sum_{j=1}^{J} u^{2}\left(\cdot, t_{j}\right)}} .
$$

Example 1 The exact solution of problem (1.1) with $f(t)=1-\cos t$ and $g(t)=0$ is given by

$$
u(x, t)=1-\frac{1}{2}\left(e^{\frac{x}{\sqrt{2}}} \cos \left(\frac{x}{\sqrt{2}}+t\right)+e^{-\frac{x}{\sqrt{2}}} \cos \left(\frac{x}{\sqrt{2}}-t\right)\right) .
$$

As the regularized solution in (4.20) is an infinite series, we compute it from $n=-50$ to $n=50$ in both examples. If we take $p=0$, the $a$ prior $i$ bound can be calculated as $E=3.1543$ according to (2.7), and the regularization parameter $\alpha=0.0054,0.0277$ from (4.9) for $\varepsilon=0.01,0.05$, respectively.

Figure 1 compares the stability of the regularized solution computed by the classic Tikhonov method and the modified Tikhonov method at fixed point $x=0.4$ with $\varepsilon=0.01$. The relative root mean square errors for them are $e(u)=0.0060$ and $e(u)=0.0059$, re-

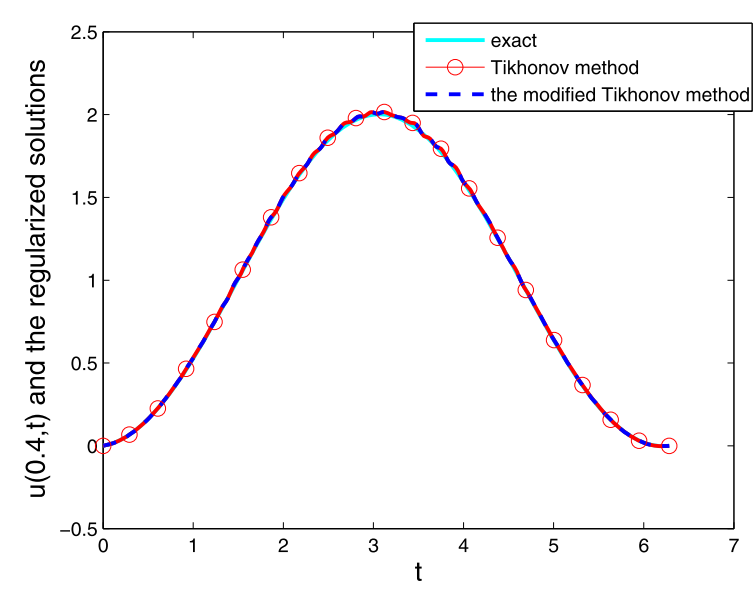

Figure 1 The Tikhonov regularized solution and the modified Tikhonov regularized solution at fixed point $x=0.4$ with $\varepsilon=0.01$. 


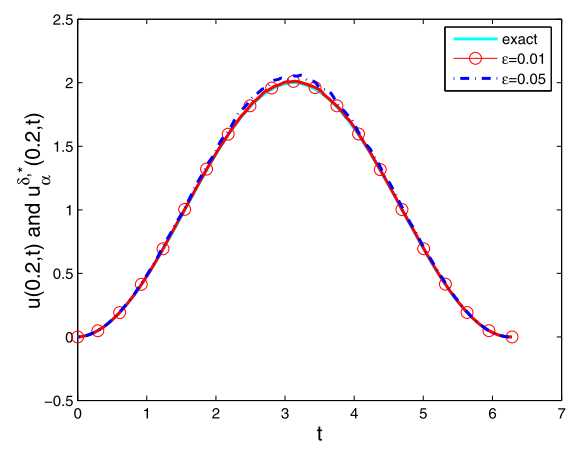

(a)

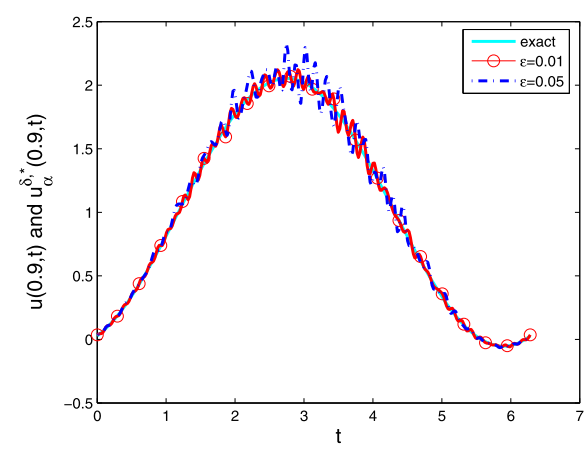

(c)

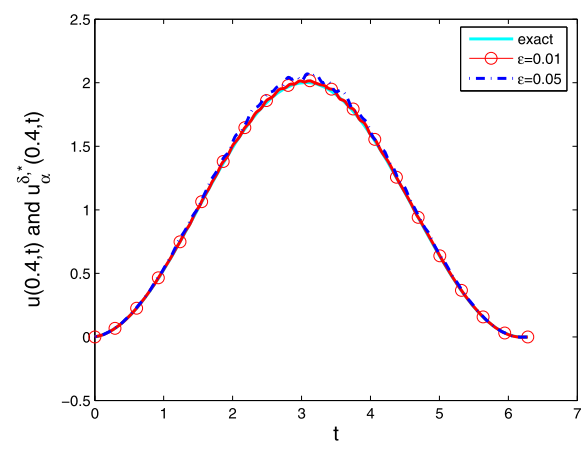

(b)

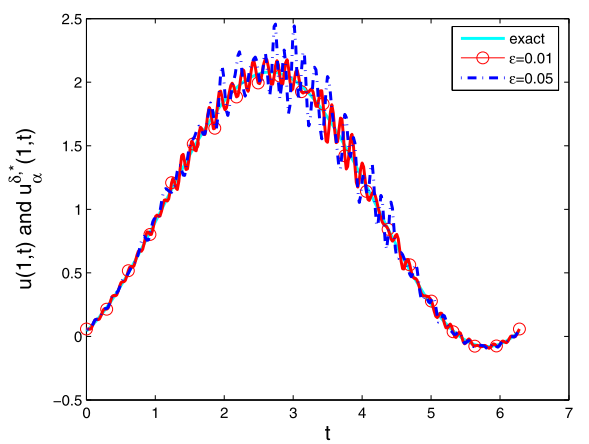

(d)

Figure 2 Comparison of the exact solution with the modified Tikhonov regularized solution. (a) $x=0.2$, (b) $x=0.4$, (c) $x=0.9$, (d) $x=1$.

Table 1 The relative root mean square errors with $\varepsilon=0.01$ and $\varepsilon=0.05$ for Example 1

\begin{tabular}{lllllll}
\hline $\boldsymbol{x}$ & $\mathbf{0 . 2}$ & $\mathbf{0 . 4}$ & $\mathbf{0 . 6}$ & $\mathbf{0 . 8}$ & $\mathbf{0 . 9}$ & $\mathbf{1}$ \\
\hline$e_{0.01}(u)$ & 0.0051 & 0.0059 & 0.0095 & 0.0198 & 0.0300 & 0.0460 \\
$e_{0.05}(u)$ & 0.0245 & 0.0254 & 0.0299 & 0.0457 & 0.0627 & 0.0899 \\
\hline
\end{tabular}

spectively. For these two methods, there is almost no difference in the numerical results. However, in theoretical analysis, it is much easier to obtain the explicit error estimate for the modified Tikhonov method than to do it for the classic Tikhonov method.

Figure 2 gives the comparison of the exact solution and its approximations with different noise. Since the exact solution $u(x, t)$ is a periodic function with variable $t$, the approximate solution converges to the exact solution everywhere. We see that the approximations are acceptable for both interior and boundary temperature, and the numerical results are stable with the increase of noisy levels.

Table 1 shows the relative root mean square errors for different $x$ with $\varepsilon=0.01$ and $\varepsilon=0.05$, respectively. From this table, it is easy to see that the smaller the $x$ the better the computed approximation. This is consistent with the theoretical result (4.21).

In the next example, we will show the case in which the exact solution is not given.

Example 2 The solution itself satisfies the following equations:

$$
u_{t}=u_{x x}, \quad 0<x<1,0<t<2 \pi,
$$




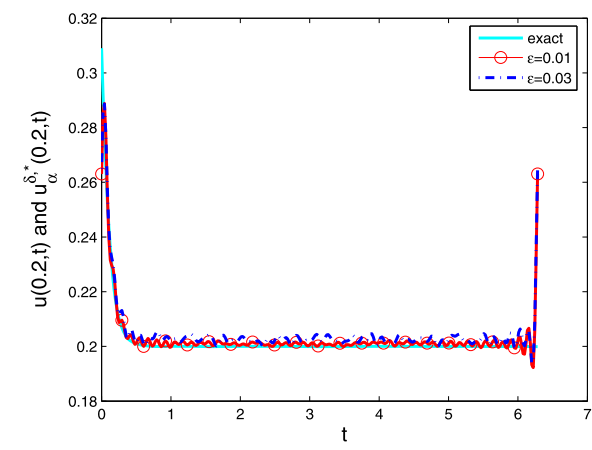

(a)

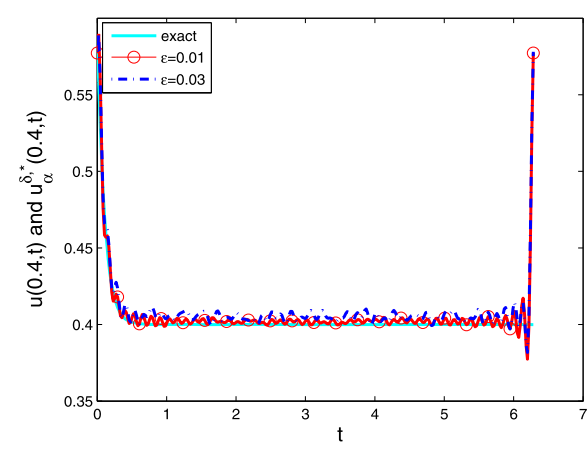

(b)

Figure 3 Comparison of the exact solution with the modified Tikhonov regularized solution. (a) $x=0.2$, (b) $x=0.4$.

$$
\begin{aligned}
& u(0, t)=0, \quad 0 \leq t \leq 2 \pi, \\
& u(1, t)=H(t), \quad 0 \leq t \leq 2 \pi,
\end{aligned}
$$

where $H(t)$ is the Heaviside function. We use the method of the fundamental solution [14] to solve the forward problem and obtain $g(t)=u_{x}(0, t)$. The boundary data $g(t)$ is disturbed by a random error, and the modified Tikhonov method is used to stabilize this inverse heat conduction problem.

For this example, we can calculate by Matlab that $E=2.5238$, and the regularization parameter $\alpha=0.0059,0.0165$ for $\varepsilon=0.01,0.03$, respectively.

In Figure 3, we see that the regularized solution is drastically oscillatory at $t=2 \pi$, while the numerical result is acceptable for other points. The reason for this phenomenon is that the solution $u(x, t)$ is not periodic to variable $t$, and thus the Fourier series (2.6) does not converge at the endpoint.

\section{Conclusion}

In this paper, the inverse heat conduction problem with only boundary value in a bounded domain has been investigated. The conditional stability is given. We propose a modified Tikhonov regularization method for obtaining a regularized solution. Based on an a priori assumption for the exact solution, the order optimal error estimate is obtained with a suitable choice of regularization parameter. Numerical examples show that our proposed method is effective and stable.

\section{Competing interests}

The authors declare that they have no competing interests.

Authors' contributions

All authors read and approved the final manuscript.

\section{Author details}

'College of Science, Henan University of Technology, Zhengzhou, 450001, P.R. China. ${ }^{2}$ School of Mathematics and Information Science, Yantai University, Yantai, Shandong 264005, P.R. China.

\section{Acknowledgements}

The authors would like to thanks the editor and the referees for their valuable comments and suggestions that improve the quality of our paper. We would like to thank Professor CL Fu for his very kind help and advice. The work is supported by the National Natural Science Foundation of China $(11171136,11561045)$ and the Natural Science Foundation of Henan Province of China (132300410231, 132300410232). 
Received: 11 February 2016 Accepted: 6 May 2016 Published online: 16 May 2016

\section{References}

1. Dorroh, JR, Ru, XP: The application of the method of quasi-reversibility to the sideways heat equation. J. Math. Anal. Appl. 236(2), 503-519 (1999)

2. Hadamard, J: Lectures on the Cauchy Problems in Linear Partial Differential Equations. Yale University Press, New Haven (1923)

3. Eldén, L: Approximations for a Cauchy problem for the heat equation. Inverse Probl. 3, 263-273 (1987)

4. Hào, DN, Reinhardt, HJ, Schneider, A: Numerical solution to a sideways parabolic equation. Int. J. Numer. Methods Eng. 50(5), 1253-1267 (2001)

5. Engl, HW, Hanke, M, Neubauer, A: Regularization of Inverse Problems. Kluwer Academic, Boston (1996)

6. Eldén, L, Berntsson, F, Regińska, T: Wavelet and Fourier methods for solving the sideways heat equation. SIAM J. Sci. Comput. 21(6), 2187-2205 (2000)

7. Regińska, T, Eldén, L: Solving the sideways heat equation by a wavelet-Galerkin method. Inverse Probl. 13(4), 1093-1106 (1997)

8. Regińska, T, Eldén, L: Stability and convergence of wavelet-Galerkin method for the sideways heat equation. J. Inverse III-Posed Probl. 8, 31-49 (2000)

9. Cheng, W, Fu, CL: Solving the axisymmetric inverse heat conduction problem by a wavelet dual least squares method. Bound. Value Probl. 2009, Article ID 260941 (2009)

10. Carasso, A: Determining surface temperatures from interior observations. SIAM J. Appl. Math. 42, 558-574 (1982)

11. Murio, DA: The Mollification Method and the Numerical Solution of III-Posed Problem. Wiley, New York (1993)

12. Murio, DA: Stable numerical evaluation of Grünwald-Letnikov fractional derivatives applied to a fractional IHCP. Inverse Probl. Sci. Eng. 17(2), 229-243 (2009)

13. Garshasbi, M, Dastour, $\mathrm{H}$ : Estimation of unknown boundary functions in an inverse heat conduction problem using a mollified marching scheme. Numer. Algorithms 68(4), 769-790 (2015)

14. Hon, YC, Wei, T: The method of fundamental solutions for solving multidimensional inverse heat conduction problems. Comput. Model. Eng. Sci. 7(2), 119-132 (2005)

15. Wróblewska, A, Frackowiak, A, Cialkowski, M: Regularization of the inverse heat conduction problem by the discrete Fourier transform. Inverse Probl. Sci. Eng. 24(2), 195-212 (2016)

16. Wang, Y, Cheng, J, Nakagawa, J, Yamamoto, M: A numerical method for solving the inverse heat conduction problem without initial value. Inverse Probl. Sci. Eng. 18(5), 655-671 (2010)

17. Ginsberg, F: On the Cauchy problem for the one-dimensional heat equation. Math. Comput. 17, $257-269$ (1963)

18. Liu, JC, Wei, T: A quasi-reversibility regularization method for an inverse heat conduction problem without initial data. Appl. Math. Comput. 219, 10866-10881 (2013)

19. Yang, F, Fu, CL: The method of simplified Tikhonov regularization for dealing with the inverse time-dependent heat source problem. Comput. Math. Appl. 60(5), 1228-1236 (2010)

20. Cheng, W, Fu, CL, Qian, Z: A modified Tikhonov regularization method for a spherically symmetric three-dimensional inverse heat conduction problem. Math. Comput. Simul. 75(3-4), 97-112 (2007)

21. Kirsch, A: An Introduction to the Mathematical Theory of Inverse Problems. Springer, New York (1996)

\section{Submit your manuscript to a SpringerOpen ${ }^{\circ}$ journal and benefit from:}

- Convenient online submission

Rigorous peer review

- Immediate publication on acceptance

- Open access: articles freely available online

- High visibility within the field

- Retaining the copyright to your article 